\title{
Evaluation of Loss Coefficient For Stand Alone Radiator
}

\author{
G.Pillutla $^{1}$, R. Mishra ${ }^{1}$, S.M.Barrans ${ }^{1}$ J.Barrans $^{2}$ \\ ${ }^{1}$ University of Huddersfield, Queensgate, Huddersfield HD1 3DH, UK \\ ${ }^{2}$ Heights - UK Limited, Halifax, UK \\ Phone/Fax number: +00447821683892, e-mail: g.pillutla@hud.ac.uk, r.mishra@hud.ac.uk
}

\begin{abstract}
In the UK, domestic heating contributes to about $40 \%$ of annual energy consumption. Effective and efficient heating systems are essential to drive the cost of heating down. Although there are several types of heating systems, radiators are the most popular heat emitters. Head loss in a radiator depends on various design parameters based on fluid flow path conditions and design of the radiator. The work presented in this paper identifies and compares the loss co-efficient for two most common configurations of radiators used in domestic heating systems. These are Bottom-Bottom Opposite Ends (BBOE) and Bottom-Top Opposite Ends (BTOE) configurations for a standalone system. In a standalone radiator design the loss co-efficient $\mathrm{K}$ value varies with the panel configuration and flow path in the BBOE and BTOE layouts. Similar to loss co-efficient in a pipe system the $\mathrm{K}$ value in a radiator system is a function of the Reynolds number. It has been found that double and single panel radiators have significantly different behaviour for the two flow layouts with higher $\mathrm{K}$ values for the BTOE configuration at lower velocity.
\end{abstract}

\section{Key words}

Flow path, Double panel, Single Panel, K value, Frictional loss

\section{Introduction}

Domestic and industrial heating systems construe a big industry in cooler countries, globally. Radiators in a central heating system have been a primary source of domestic heating in the UK for several decades. In a typical central heating system, water is heated at a "central node" (boiler) and is then pumped through the pipe work to individual radiators. The radiators act as heat exchangers and heat the ambient air. This system has several drawbacks which include little flexibility, limited controllability and poor expandability.

A stand alone system overcomes the drawbacks of a central heating system by having closer monitoring and control of heat and flow. Such a system has a reduced number of convector fins which are replaced by a metal heating element and a pump to circulate the water within a closed loop. A controller with a thermostat on the heating element is used to regulate the temperature of water/fluid. A radio frequency remote thermostat monitors the room temperature and feeds back to the control board in the radiator. Each unit is self contained and may be operated individually or as part of groups of radiators.

Radiators have been analyzed for their performance by various researchers. Peach, Walters and Ward [1]-[3] have found that the aspect ratio (length vs. height) of the radiator has a direct influence on radiator heat output. About 30- $50 \%$ of the radiator heat output is emitted by radiation and the remainder through convection. Emissivity of the surface affects the performance and studies recommend an oxidized metallic surface for best performance.

Beck et. Al, [4] have carried out extensive investigation to analyze the working of radiator panels in a central heating system. They have reported that the output of radiators can be increased by optimizing the location of the radiators within the room. Also decreasing the height above the ground and by increasing their spacing from the wall would improve the air flow characteristics over the radiator. The attachment of convector fins to panel radiators increases the surface area and hence the convective heat transfer. They also concluded that different combinations of fluid entry and exit positions can affect radiator performance. Peach [1] observed that introducing the flow at the top and exit at the bottom on the opposite end (TBOE) can improve the temperature distribution within the radiator in a central heating system; this would perform better than the Bottom Bottom Opposite Ends (BBOE) configuration. This layout is currently used in standard installations. Hot water introduced at the bottom of the radiator influences the flow pattern by rising to the top due buoyancy. Gravity also influences the flow pattern. The point of entry and exit are hence critical to achieve maximum temperature drop in the fluid, consequently increasing the amount of heat transferred.

Ward's [3] work has identified that the as the flow rate is reduced the residence time of water in the radiator increases resulting in lower return temperature. McIntyre [5] further concluded that the heat output of a radiator decreases with a decrease in flow rate of water; while Giesecke [7] has found that in a central heating radiator system frictional head loss increases with the increase in flow rate. Design of the system has implication on the thermal output of the system. The flow rate in a given 
system influences its heat output and hence a suitable pump should be selected to meet the pumping demand for maximum heat output whilst accounting for the head loss in the system.

A combination of flow rate, temperature drop and mixing of fluid in the radiator make it difficult to predict the output of the radiator in practice particularly in conjunction with thermostatic control valves. Due to use of a dedicated pump the flow rate within a radiator in standalone systems is higher than that in conventional systems. This results in mixing. Unlike a central heating system, a combination of thermistor and room air temperature sensor for each radiator, maintains the inlet temperature within $+/-3$ deg $\mathrm{C}$. This influences the flow path of the water/fluid. Previous work by Pillutla [7] on the effect of point of entry on the thermal performance of radiators suggests that a BTOE layout produces a uniform temperature distribution and maximum temperature drop. The present work aims to develop an empirical relationship between the pipe configuration geometry and the head loss coefficient for a standalone radiator system. This relationship would help increase the understanding of characteristics of a standalone radiator system, optimise its flow and consequently help reduce the required pumping power for maximum heat emission.

\section{EXPERIMENTAL SETUP RANGE OF PARAMETERS}

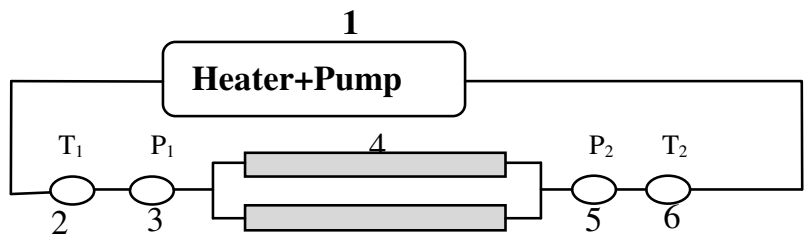

Figure 1: Experimental Setup

In the present investigation a number of studies have been carried out experimentally. For this purpose both double panel and single panel radiators with different combinations of point of fluid entries were used as a standalone system. Both the radiators were $300 \mathrm{~mm}$ high and $600 \mathrm{~mm}$ long. They were sourced from a single supplier to eliminate the effect of material grade, profile, end connector size and shape.Table 1 lists various parameters that have been measured or computed in the present investigation. Figure 1 shows the schematic diagram of the experimental setup. The diagram shows (1) control unit comprising heater element control electronics and a circulating pump. The outlet of the pump is connected to the bottom left of the radiator (4), this point would be referred to as the inlet point and is common for both the layouts. For the first configuration (BBOE) the outlet of the radiator is at the bottom right of the radiator. Water is filled using the top left entry point. The top right point is fitted with an air bleed valve to ensure the radiator is completely filled with water. For a BTOE configuration the outlet is at the top right of the radiator with the bleed valve located at the top left. The water is filled at bottom right for BTOE layout. To evaluate the flow performance digital pressure gauges (3) and (6) were used at inlet and outlet to measure the. A ball valve is used to control the flow rate of water in the system. A flow meter is used between the outlet of the pump and the inlet of the radiator to determine the flow rate. To evaluate the thermal performance K-type thermocouples (2) and 5 were used to measure the inlet and outlet temperatures of water. For the purpose of the study the temperature has been fixed at $70 \mathrm{deg} \mathrm{C}$ and the performance is evaluated at flow rates corresponding to two valve position (100\% and 50\%). An eight channel 'Squirrel Data Logger' is used to log the information at $10 \mathrm{sec}$ intervals.

\begin{tabular}{|c|c|}
\hline Symbol & Description \\
\hline$H_{f}$ & Total head loss \\
\hline$\Delta P$ & $\begin{array}{c}\text { Pressure difference } \\
\text { between inlet and } \\
\text { outlet of radiator }\end{array}$ \\
\hline$\rho$ & $\begin{array}{c}\text { Density of fluid } \\
\text { Gravitational } \\
\text { acceleration } \\
\text { Mass flow rate }\end{array}$ \\
\hline$g$ & Constant \\
\hline$V$ & $\begin{array}{c}\text { Co-efficient of surface } \\
\text { friction }\end{array}$ \\
\hline $\mathrm{k}$ & Hydraulic diameter \\
\hline$f_{c}$ & Reynolds Number \\
\hline$D_{H}$ & Dynamic viscosity \\
\hline$R_{e}$ & Area of cross section \\
\hline$\mu$ & Perimeter of cross \\
\hline $\mathrm{A}$ & section \\
\hline $\mathrm{P}$ &
\end{tabular}

Table 1: Description of parameters

\begin{tabular}{|l|l|}
\hline \multicolumn{1}{|c|}{ Parameter } & \multicolumn{1}{c|}{ Range } \\
\hline $\mathrm{P} 1 \rightarrow$ Inlet pressure & $0.64-2.98 \times 10^{5} \mathrm{~Pa}$ \\
\hline $\mathrm{P} 2 \rightarrow$ Outlet pressure & $0.47-2.42 \times 10^{5} \mathrm{~Pa}$ \\
\hline $\begin{array}{l}\mathrm{V} \rightarrow \text { Mass flow rate of } \\
\text { water }\end{array}$ & $2.76-6.74 \times 10^{-5} \mathrm{~m}^{3} / \mathrm{s}$ \\
\hline
\end{tabular}

Table 2: Description of Parameters

To start the experiment the rig was setup for the required configuration (BBOE or BTOE). The experiments were done in a temperature controlled environment to ensure maximum thermal load on the system. Once the room temperature was stabilized the thermocouples, flow meter and pressure sensors were connected to the computer via the data logger. The operating temperature of the radiator was set using a radio frequency controller. The experiments were conducted using a mechanical valve and the flow rate of the system was to $50 \%$ and $100 \%$ valve opening position. The data logger software and a thermal camera were set to capture the readings and images at $10 \mathrm{sec}$ interval. The thermal camera captures the flow of hot fluid within the radiator. The images are 
used to quantify the radiator surface temperature and help visualize the fluid path. The radiator was now turned on and left running for 60 minutes. The data for the present study was used after the system reached a steady state.

Head loss in standalone alone system can be mainly attributed to loss at entry, loss in the radiator panel and loss at the exit. Where, loss in the radiator is a combination of frictional loss and the complexity of fluid path for the given condition. To capture the effect of inlet and outlet connector and the radiator, care was taken that theta the pressure gauge 3 was located at the upstream of inlet connection and gauge 6 at the downstream outlet connector and flow control valve. For a set valve position, it has been observed that there is approximately $10 \%$ variation in the flow velocity. Head loss in a system is computed by equation 1 . It is very difficult to determine the surface friction co-efficient for the radiator due to the complexity of the geometry and access to the flow path. Hydraulic diameter is typically calculated by using equation 5. Due to wide range of path length within the radiator for different pipe layouts and panel configuration, hydraulic diameter of the inlet pipe has been used as for the study. ' $\mathrm{K}$ ' is a constant for the system under consideration dependant on friction coefficient of system and hydraulic diameter.

$$
H_{f}=f_{c} D_{H} \frac{V^{2}}{2 g}=K \frac{V^{2}}{2 g}=\frac{\Delta P}{\rho g}
$$

Where

$$
K=F\left(f_{c}, D_{H}\right)
$$

Therefore

$$
\begin{aligned}
& K=\frac{H_{f} \times 2 g}{V^{2}}=\frac{2 \times \Delta P}{\rho V^{2}} \\
& R_{e}=\frac{\rho V D_{H}}{\mu} \\
& D_{H}=\frac{4 A}{P}
\end{aligned}
$$

\section{RESULTS AND DISCUSSIONS}

The pressure measured at the inlet and outlet of the radiator was used to compute the pressure drop, which in turn was used to compute the loss co-efficient based on equation 2. The following results compare the nondimensional loss co-efficient for different panel and pipe configurations. Loss co-efficient against velocity has been illustrated in figure 2 to study the trend for the two pipe layouts in a single panel radiator. BBOE and BTOE configurations have similar trends, where the value for the loss co-efficient $\mathrm{K}$ drops with the increase in velocity.
BTOE configuration has a higher loss co-efficient than $\mathrm{BBOE}$ at lower flow rate. $\mathrm{K}$ at $50 \%$ flow rate (velocity of $0.25 \mathrm{~m} / \mathrm{s}$ ) for a single panel radiator in a BBOE layout was found to be 475.8 and 508.49 for a BTOE layout. The loss co-efficient was found to be 262.69 and 276.2 at $100 \%$ flow rate (velocity of $0.33 \mathrm{~m} / \mathrm{s}$ ) for BTOE and $\mathrm{BBOE}$ configurations respectively. The change in the loss co-efficient in a BTOE configuration is 245.96 which are higher than BBOE at 199.6. As shown in figure 3 the two double panel radiators have very similar trend for the two pipe configurations, with the slopes varying within $3 \%$ of each other. Nevertheless the trend in a double panel radiator is more gradual as compared to a single panel type. Similar to the single panel radiator the loss co-efficient $(\mathrm{K})$ is higher in a BTOE configuration than in BBOE configuration.

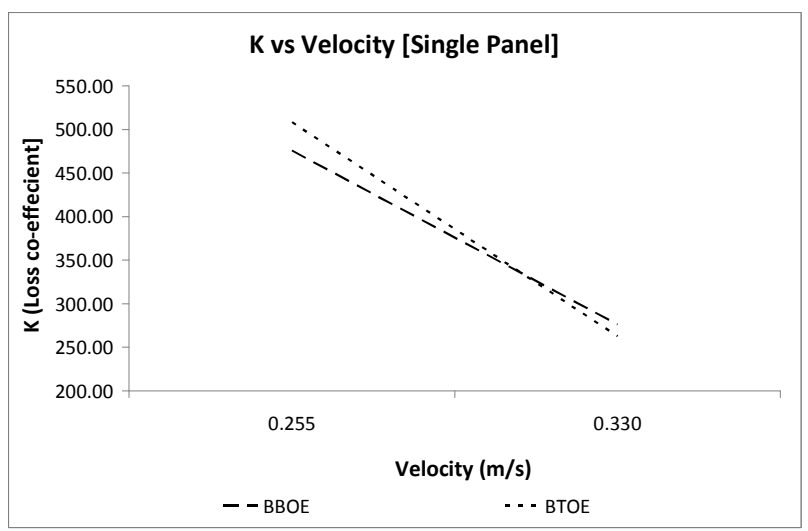

Figure 2: Constant $k$ as a function Velocity [Single Panel]

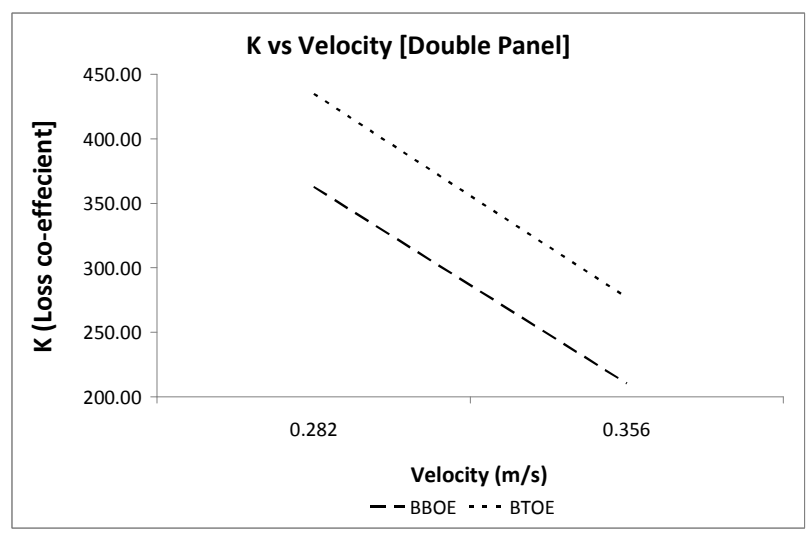

Figure 3: Constant $k$ as a function Velocity [Double Panel]

Loss co-efficient at $50 \%$ flow rate (velocity of $0.28 \mathrm{~m} / \mathrm{s}$ ) for a double panel radiator in a BBOE layout is 362.79 ; and it is 434.92 for a BTOE layout. The loss co-efficient is 276.88 and 210.29 at $100 \%$ (velocity of $0.36 \mathrm{~m} / \mathrm{s}$ ) flow rate for BTOE and BBOE layouts respectively. The difference in loss coefficients in the two radiators and pipe layouts can be attributed to different flow path within the radiator. This is further supported by thermal images for the respective flow conditions shown in table 3. In a BBOE layout for the both radiator designs a portion of the hot water rises up and flows across the top after it enter the radiator at the bottom left corner. The 
remaining water flows across the bottom towards the exit point of the radiator. The two systems show differences as the flow develops, and the path becomes more complicated.

After the hot water enters the radiator in the BTOE configuration there is an equal distribution of flow near the top of the radiator and across its bottom. The water tends to rise in the single panel radiator. Similar to the $\mathrm{BBOE}$ layout the path gets complex as the flow develops further.

For a straight pipe the frictional co-efficient is expressed by equation 5 [8], where $\mathrm{A}$ is constant for a system depending on fouling. Loss co-efficient, being a function of frictional co-efficient and hydraulic diameter, can effectively be expressed as a function of the Reynolds number. The effect of pipe configuration on the correlation has been evaluated for a double panel radiator in figure 4.

$f_{c}=\frac{A}{\operatorname{Re}^{-0.145}}$

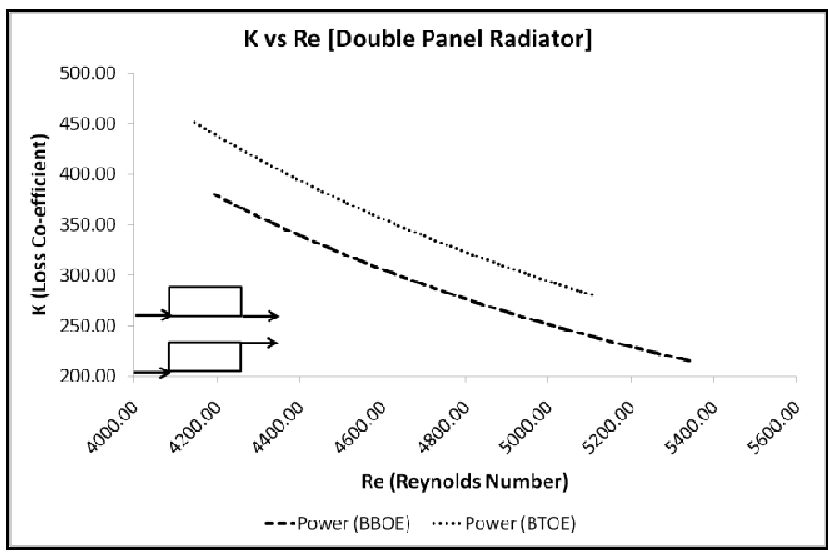

Figure 4: Loss Co-efficient Constant vs Reynolds Number [Double Panel Radiator]

As indicated by the curves in fig.4, the loss coefficient in the radiator decreases with an increase in Reynolds number for both pipe layouts investigated. In the BBOE configuration the loss co-efficient is 362.79 at 4224 Reynolds number and 210.29 at 5341 Reynolds number. The loss co-efficient for a double panel BBOE configuration can be expressed as a function of Reynolds number by equation (6).

$K=\frac{1 \times 10^{11}}{\operatorname{Re}^{2.325}}$

The loss co-efficient in the BTOE configuration is greater than in the BBOE layout with the $\mathrm{K}$ value of 434.92 at $50 \%$ flow rate and 276.88 at $100 \%$ flow rate. Similarly BTOE configuration in a double panel radiator can be expressed by equation (7).

$$
K=\frac{6 \times 10^{10}}{\operatorname{Re}^{2.239}}
$$

On comparing equation 6 and 7 it can be observed that the constant $\mathrm{A}$ for the double panel $\mathrm{BBOE}$ configuration is $1 \times 10^{11}$, while in a double panel BTOE layout it is $6 \times 10^{10}$.

The exponent values for the Reynolds number are 2.325 (eq.6) and 2.239 (eq.7) for the BBOE and BTOE layouts. These two values differ by only $3.7 \%$, further confirming that the two layouts have very similar trends in a double panel radiator.

Figure 5 illustrates loss co-efficient as a function of Reynolds number for a single panel radiator. Contrary to the double panel radiator the two pipe layouts do not have similar trends. BBOE layout has a gradual drop in the loss co-efficient with $\mathrm{K}$ value of 475.8 at $50 \%$ flow rate and a Reynolds number of 3821 . At $100 \%$ flow rate the $\mathrm{K}$ value for the $\mathrm{BBOE}$ configuration is 276.2 and is 262.69 for the BTOE configuration. The power curves for the two pipe layouts cross over at $\mathrm{K}$ value of 370 and Reynolds number of 4250. Equation 8 and 9 give the relation between the $\mathrm{K}$ value and Reynolds number for the single panel BBOE and BTOE layout respectively.

$$
\begin{gathered}
K=\frac{2 \times 10^{10}}{\operatorname{Re}^{2.096}} \\
K=\frac{4 \times 10^{11}}{\operatorname{Re}^{2.5}}
\end{gathered}
$$

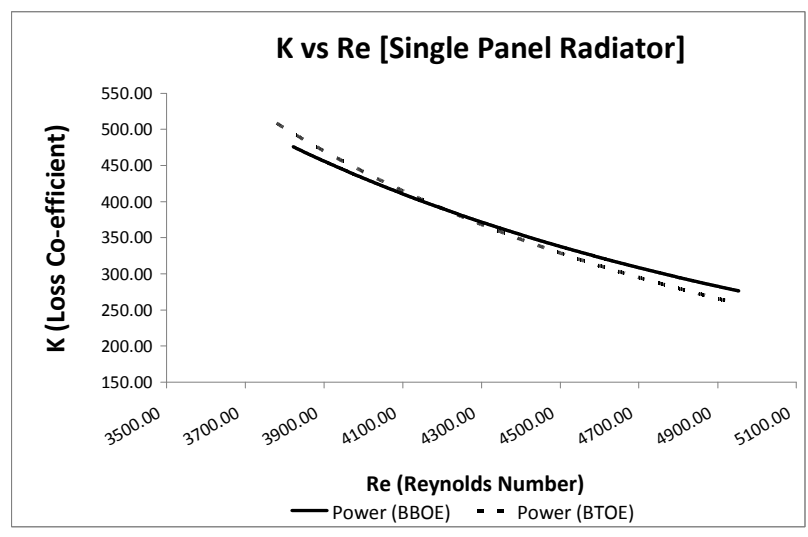

Figure 5: Loss Co-efficient Constant vs Reynolds Number [Single Panel Radiator]

The constant A for the single panel BBOE configuration is $2 \times 1010$, while in a single panel BTOE configuration it is $4 \times 1011$. 


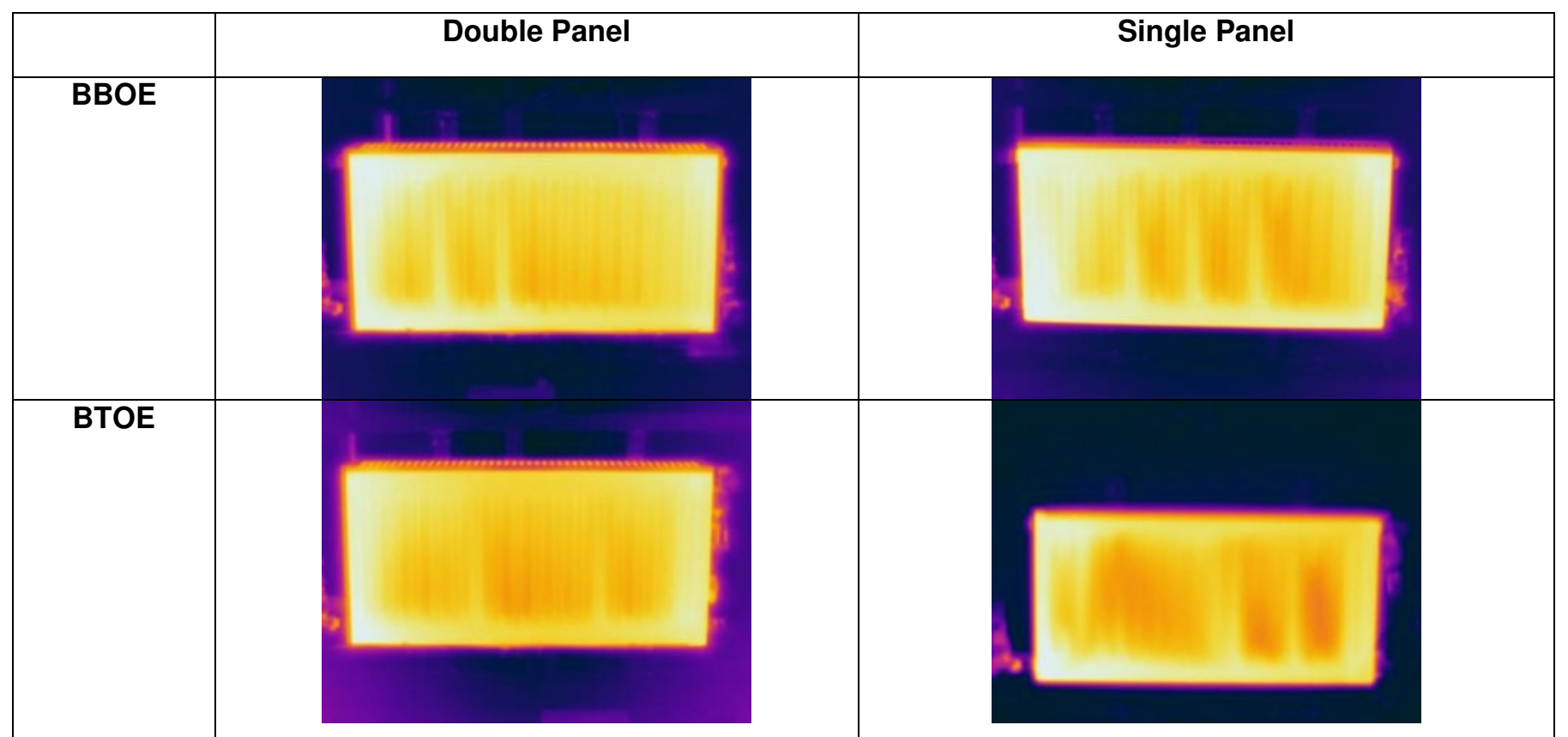

Table 3: Thermal Images for single and double panel radiators in different pipe layout

The exponent for the BBOE configuration is 2.096, resulting in a steep curve compared to a double panel BBOE configuration. The exponent for the single panel BTOE layout is 2.5 which makes the curve gradual than the equivalent double panel radiator.

\section{CONCLUSION \& FURTHER WORK}

Loss co-efficient for a $300 \mathrm{~mm} \times 600 \mathrm{~mm}$ double and single panel radiator have been calculated for two flow configurations in a standalone system. The loss coefficient and hence the frictional loss in the standalone system drops with the increase in the water velocity. A double panel radiator with $\mathrm{BBOE}$ layout has the lowest loss co-efficient suggesting it would require least pumping power. Previous work has suggested that a BTOE layout has maximum temperature drop and hence better heat output. For this configuration, flow velocity can be optimised for low $\mathrm{K}$ value and maximum heat output with minimal pumping power. The present study has also established a correlation between the $\mathrm{K}$ values and Reynolds number, which is a function of velocity and hydraulic diameter. This study can be used to reduce the pumping power and hence the pump size in the current design. Further work is proposed to account for radiator size and quantify thermal dependence of the loss co-efficient. The work could be further supported by testing a transparent radiator with LDV (Laser Doppler Velocitymetery) system to quantify the fluid path.

\section{Acknowledgement}

The project was initiated under the KTP program between Heights UK Limited and University of Huddersfield. Many thanks to company to permit us to continue our research on the product and publish information even after completion of the program in 2008.

\section{References}

[1] J Peach "Radiators and other Convectors" (1972), Environmental science and technology vol 39 pp 239-253

[2] K Walters and RH Fine "The Performance of Radiators and Convectors Using Medium Temperature Hot Water Laboratory report" (1973) Heating and ventilation research association

[3] IC Ward "Domestic Radiators: Performance at lower mass flow rates and lower temperature differentials than those specified in standard performance test" (1991) Building service engineering research and technology (12) pp 87-94

[4] SMB Beck; SC Grinsted; SG Blackey; K Worden K "A novel design for panel radiators" (2004) Applied Thermal Engineering (24) pp 1291-1301

[5] DA McIntyre "Output of radiators at reduced flow rate" (1986) Building Services Engineerng Reaserch and Technology vol 7 (2) pp 92-95

[6] FE Giesecke; AP Kratz "Heat emissions and friction heads of hot water radiators and convectors" (1945) University of Illinois Urbana

[7] G Pillutla; R Mishra; SM Barrans; J Barrans "Experimental Investigation of Effect of Point of Fluid Entry in Standalone Domestic Heating Radiator" (2009) Fluid Mechanics and Fluid Power conference Pune

[8] DS Miller "Internal Flow- A guide to losses in pipe and duct systems" (1971) The British hydromechanics research association, Cranfield UK 